\title{
Bead Size Effects on Protein-Medlated DNA Looping in Tethered-Particle Motion Experiments
}

\author{
J. N. Milstein, ${ }^{1}$ Y. F. Chen, ${ }^{2}$ J.-C. Meiners ${ }^{1,3}$ \\ ${ }^{1}$ Department of Physics, University of Michigan, Ann Arbor 48103 \\ ${ }^{2}$ Department of Biomedical Engineering, University of Michigan, Ann Arbor 48103 \\ ${ }^{3}$ Biophysics in the College of Literature, Science, and the Arts, University of Michigan, Ann Arbor 48103
}

Received 17 May 2010; revised 21 September 2010; accepted 21 September 2010

Published online 29 September 2010 in Wiley Online Library (wileyonlinelibrary.com). DOI 10.1002/bip.21547

\section{ABSTRACT:}

Tethered particle motion (TPM) has become an important tool for single-molecule studies of biomolecules; however, concerns remain that the method may alter the dynamics of the biophysical process under study. We investigate the effect of the attached microsphere on an illustrative biological example: the formation and breakdown of protein-mediated DNA loops in the lac repressor system. By comparing data from a conventional TPM experiment with $800 \mathrm{~nm}$ polystyrene beads and dark-field TPM using $50 \mathrm{~nm} A u$ nanoparticles, we found that the lifetimes of the looped and unlooped states are only weakly modified, less than twofold, by the presence of the large bead. This is consistent with our expectation of weak excluded-volume effects and hydrodynamic surface interactions from the cover glass and microsphere. (C) 2010 Wiley Periodicals, Inc. Biopolymers 95: 144-150, 2011.

Keywords: DNA looping, single molecule techniques, TPM

This article was originally published online as an accepted preprint. The "Published Online" date corresponds to the preprint version. You can request a copy of the preprint by emailing the Biopolymers editorial office at biopolymers@wiley. com

Correspondence to: Joshua Milstein; e-mail: milsteij@umich.edu Contract grant sponsor: National Institutes of Health

Contract grant number: GM 065934

(C) 2010 Wiley Periodicals, Inc.

\section{INTRODUCTION}

ethered particle motion (TPM) is a simple and increasingly popular experimental technique to study protein-DNA interactions at the single molecule level. In TPM, the biomolecule of interest is attached at one end to a microscope cover glass and at the other end a marker, typically a polystyrene microsphere, is affixed. ${ }^{1}$ The motion of the marker bead is observed by standard light microscopy (i.e., bright-field, dark-field, differential interference contrast, or fluorescence $)^{2}$ to infer dynamical information about the biomolecular system. Typical examples are protein binding and dissociation rates or velocities of motor molecules. $^{3-5}$ While such experiments can be performed with small markers such as gold nanoparticles, in practice, a larger marker microsphere with a radius of several hundreds of nanometers is often preferred because good image quality can be readily achieved with an inexpensive and robust bright-field microscopy set-up. Imaging nanoparticles, on the other hand, requires cumbersome dark-field imaging and an expensive low-light camera. ${ }^{6}$ When the marker bead is as large as in conventional TPM, often comparable to or even larger than the contour length of the molecule under study, a common concern is that the presence of such a large bead might affect the dynamics of the system of interest to the point where measured rate constants or velocities may no longer accurately reflect those of the native system. To justify the utility of TPM with conventional microspheres as an effective and quantitatively accurate assay, we provide an illustrative example of a biological process, one that should be quite sensitive to bead-induced effects, and show that the method only weakly affects the underlying biophysical behavior and measured rate constants.

Potential sources of such distorting bead effects are excluded-volume interactions between the bead and the biomolecule or cover slip, noise that is introduced into the 
system by the Brownian motion of the bead, and hydrodynamic interactions between the bead, biomolecule and cover slip. In this article, we directly assess the severity of these bead-induced effects on the rate constants of a biological process, namely the formation and breakdown rates of lac repressor ( $\mathrm{LacI}$ ) mediated DNA loops. This system was chosen because it is both representative of a typical TPM experiment and because the loop formation process has been shown to be acutely sensitive to tension in the DNA, ${ }^{7}$ which might arise as an entropic excluded-volume effect from attachment to a large microsphere. ${ }^{8}$

To quantify bead effects, we have performed a modified TPM experiment that uses Au nanoparticles of only $50 \mathrm{~nm}$ in diameter and compared the results to data from a more conventional TPM experiment that uses $800 \mathrm{~nm}$ polystyrene microspheres. For our purposes, the Au particles allow us to minimize bead effects, to get as close as possible to studying the LacI mediated looping of DNA without probe induced artifacts. Our experimental results show that bead size effects only contribute about a factor of 2 to the rate of loop formation, further validating TPM as a valuable quantitative method for studying dynamic biological processes.

\section{RESULTS}

$\mathrm{Au}$ nanoparticle and conventional TPM experiments where performed to test for bead effects on the rates of DNA association and dissociation as a typical biophysical application of TPM. The extracted dwell times for the looped and unlooped states are pooled as cumulative probability distributions as shown in Figure 1. The loop breakdown data was well fit by a single exponential function of the form

$$
P(t: \tau)=1-e^{t / \tau}
$$

with lifetime $\tau$. The loop formation data, however, could not be fit by a single exponential, but was well fit by a double exponential function. This has been observed previously ${ }^{4-5}$ and is the result of there being a multiplicity of available protein occupancy configurations comprising the unlooped state. We, therefore, fit the loop data with the following biexponential function:

$$
P_{2}\left(t: \tau_{1}, \tau_{2}\right)=c P\left(t: \tau_{1}\right)+(1-c) P\left(t: \tau_{2}\right)
$$

with lifetimes $\tau_{1}$ and $\tau_{2}$ and a dimensionless weight constant c. The fits are displayed in Figure 1. Loop formation and breakdown are both faster when the Au nanoparticle is used instead of the microsphere. The effect is small for the loop formation process, and amounts to a factor of about two in

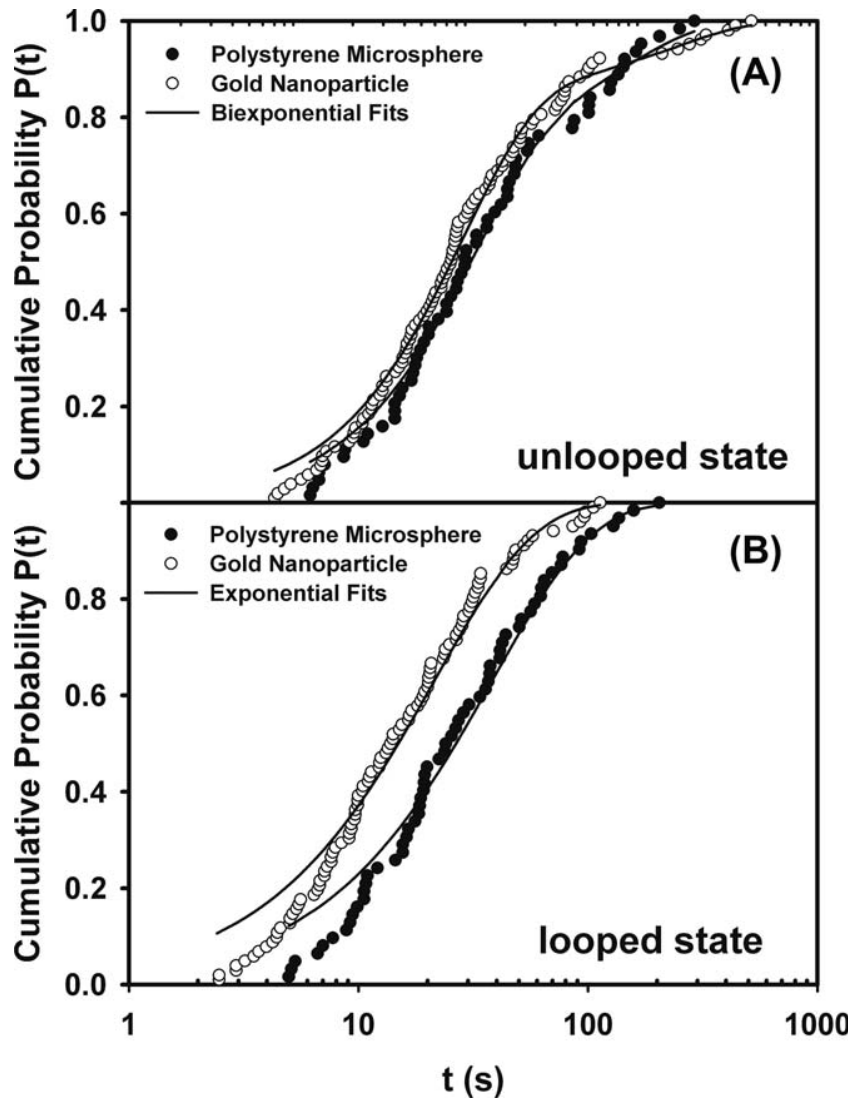

FIGURE 1 The data of (A) the unlooped state (loop formation) and (B) the looped state (loop dissociation) are fit to the biexponential function of Eq. (2), and the single exponential function of Eq. (1), respectively. For the unlooped state $\tau_{1}=8.6 \pm 0.4 \mathrm{~s}, \tau_{2}=$ $150 \pm 70 \mathrm{~s}$ and $c=0.85 \pm 0.03$ for the Au nanoparticles and $\tau_{1}=$ $10 \pm 1 \mathrm{~s}$, and $\tau_{2}=80 \pm 40 \mathrm{~s}$ and $c=0.75 \pm 0.09 \mathrm{~s}$ for the polystyrene microspheres. For the looped state $\tau=21.5 \pm 0.7 \mathrm{~s}$ for the $\mathrm{Au}$ nanoparticles and $\tau=38 \pm 2 \mathrm{~s}$ for the polystyrene microspheres.

the loop breakdown rate. For short time-scales, of only several seconds, the loop dissociation fits tend to overestimate the data. This might arise as an artifact of our $2 \mathrm{~s}$ time window; however, we have found that the fits are relatively insensitive to these points. When the fast looping events are removed between trials, the resulting fits still fall within the error bars of those presented.

The relatively good agreement between the different experiments is particularly noteworthy because they were conducted in very different regimes of coupling between the DNA motion and the microsphere motion: the dynamics of the DNA-particle construct were weakly coupled in the nanoparticle case, whereas the dynamics are strongly coupled in the polystyrene microsphere experiment. To distinguish these two regimes, Segall et al. ${ }^{8}$ introduced a dimensionless parameter, the "excursion number," which is essentially the ratio of the friction coefficients of the bead to that of the DNA, 
Table I Rate Constants From the Kinetic Model for Au Nanoparticle and Polystyrene Microsphere Marker Beads

\begin{tabular}{lrr}
\hline & Au & Polystyrene \\
\hline$k_{+} \times 10^{3}(1 / \mathrm{s})$ & $48 \pm 6$ & $45 \pm 4$ \\
$k_{-} \times 10^{3}(1 / \mathrm{s})$ & $262 \pm 7$ & $196 \pm 6$ \\
$k_{L} \times 10^{3}(1 / \mathrm{s})$ & $61 \pm 9$ & $48 \pm 5$ \\
$k_{U} \times 10^{3}(1 / \mathrm{s})$ & $40 \pm 10$ & $24 \pm 5$ \\
\hline
\end{tabular}

$N_{\mathrm{R}} \equiv R_{\mathrm{B}} /\left(l_{\mathrm{c}} l_{\mathrm{p}} / 3\right)^{1 / 2}$, where $R_{\mathrm{B}}$ is the radius of the bead, $l_{\mathrm{c}}$ is the contour length of the DNA, and $l_{\mathrm{p}}$ is the persistence length of the DNA. For the Au nanoparticles, the excursion number is $N_{\mathrm{R}}=0.3$ so the dynamics is mostly DNA-dominated, whereas $N_{\mathrm{R}}=6.0$ for the polystyrene microspheres indicates bead-dominated motion.

\section{DISCUSSION}

\section{Kinetic Model}

The physical significance of the double exponential function given by Eq. (2) becomes clearer if we consider the kinetics of loop formation. For our experiment, the process of loop formation is complicated by the presence of different unlooped substates that we are unable to distinguish. The simplest kinetic scheme that is in agreement with our data may be written as follows:

$$
S_{2 \underset{k-}{\stackrel{k_{+}}{\longrightarrow}}}^{\stackrel{k^{\prime}}{\longrightarrow}} S_{1} \stackrel{k_{L}}{\longrightarrow} L
$$

which we have previously discussed in Chen et al. ${ }^{7}$ Briefly, $S_{1}$ is the state of one vacant and one occupied operator and may directly convert to the looped state $L$ at a rate $k_{\mathrm{L}}$, or remain unlooped and convert to state $S_{2}$ at a rate $k_{-}$. State $S_{2}$, however, is an alternate configuration with both or neither operator occupied by a protein, which is not able to directly form a loop, but may convert to state $S_{1}$ at a rate $k_{+}$. This kinetic model may be solved for the time dependent probability of forming a loop

$$
L(t)=1-\frac{1}{2 \alpha}\left[\left(\kappa-\kappa_{\mathrm{L}}+\alpha\right) e^{-t / \tau_{1}}-\left(\kappa-\kappa_{\mathrm{L}}-\alpha\right) e^{-t / \tau_{2}}\right],
$$

where $k=k_{+}+k_{-}, \alpha=\left[\left(k+k_{\mathrm{L}}\right)^{2}-4 k_{+} k_{\mathrm{L}}\right]^{1 / 2}$, and the time constants are defined as $\tau_{1}=2 /\left(k+k_{\mathrm{L}}-\alpha\right)$ and $\tau_{2}=$ $2 /\left(k+k_{\mathrm{L}}+\alpha\right)$. With this result, we can unambiguously extract the four rate constants in our kinetic model (see Table I). Despite variations in protein concentration and the design of the DNA constructs, the rate constants we find are in logarithmic agreement with similar experiments of this nature. $^{5,9}$

We should note that our interest in this article is not to explore the effects of protein concentration on loop formation kinetics, as other groups have already carefully performed this study for constructs similar to the ones we employed here. ${ }^{10}$ However, as a consistency check to the model, we have performed TPM experiments with large marker beads at $1 / 5 \times$ $(40 \mathrm{pM})$ and $5 \times(1000 \mathrm{pM})$ concentrations. At $40 \mathrm{pM}, k_{\mathrm{U}}, k_{\mathrm{L}}$, and $k_{+}$remain approximately constant while $k_{-}$decreases by roughly a factor of 4 . At $1000 \mathrm{pM}$, loop formation is substantially reduced, indicating that the operators approach saturation. This suggests that at the $200 \mathrm{pM}$ protein concentration we worked with, $S_{2}$ is most likely dominated by a population of molecules with dual operator occupancy. Regardless, the exact makeup of $S_{2}$ has no bearing on our model.

If we think of the looping process as equivalent to diffusion over a potential barrier, we may associate changes to the kinetic rates as resulting from either diffusion effects, which modify the diffusion constant, or potential effects, which alter the thermodynamic energy landscape. The rates $k_{+}, k_{-}$ are independent of the potential barrier; however, they are dependent upon diffusion variables such as the total protein concentration and hydrodynamics.

The looping, $k_{\mathrm{L}}$, and unlooping, $k_{\mathrm{U}}$, rates, which can now be extracted from the data and are of primary interest to us here, may depend on both potential and diffusion effects. The potential effects are apparent if we look at the distribution histograms, such as the one in Figure 2. In equilibrium, $K=$ $n_{\mathrm{L}} / n_{\mathrm{U}}=k_{\mathrm{L}} / k_{\mathrm{U}}$, where $n_{\mathrm{U}}$ and $n_{\mathrm{L}}$ are the unlooped and looped
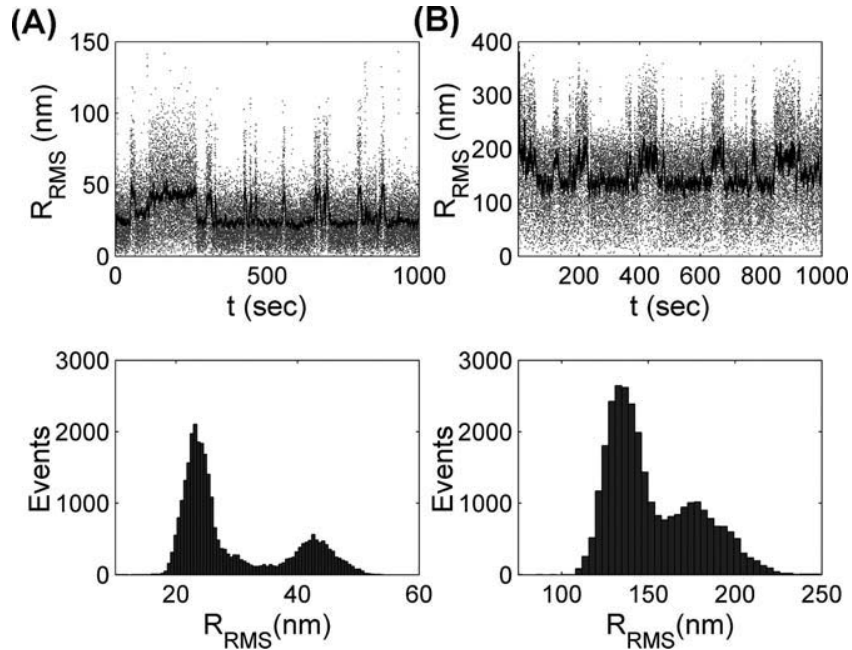

FIGURE 2 Looping data for (A) Au nanoparticles and (B) polystyrene microspheres. The uppermost plots show the root mean square displacements as a function of time. The black lines result from averaging over a moving window of $2 \mathrm{~s}$. The lower plots are histograms of the averaged motion showing two distinct states. 
population, respectively. However, $K^{\mathrm{Au}} / K^{\mathrm{PS}} \approx 2 / 3$, which shows that the equilibrium constants between the two cases, Au nanoparticles, $K^{\mathrm{Au}}$, and polystyrene microspheres, $K^{\mathrm{PS}}$, differ. Diffusion should not affect the equilibrium properties, so this is a clear sign that potential effects are important for understanding bead size effects on TPM. This, of course, does not prove that diffusive effects are irrelevant to the kinetics of the system, which is a possibility we will return to. We first focus on enumerating the various contributions that might modify the potential landscape of the system.

\section{Entropic Boundary Effects}

To understand the comparatively subtle differences observed between the two experiments, possible ways in which the probe may affect the dynamics of protein-mediated DNA loop formation and breakdown need to be considered. For instance, excluded-volume effects arise from the impenetrability of the bead and the cover glass. These geometric constraints give rise to an effective repulsive force between the bead and the cover glass, which in turn can affect loop formation and breakdown rates. An analytic form for this volume exclusion force, ${ }^{8}$ treating the DNA as a Gaussian chain and constraining the motion of the bead, but not the DNA, is given by

$$
F_{\text {eff }}=\frac{k_{\mathrm{B}} T}{\sqrt{\pi l_{\mathrm{c}} l_{\mathrm{p}} / 3}}\left(\frac{1-e^{-N_{\mathrm{R}}^{2}}}{\operatorname{erf}\left(\mathrm{N}_{\mathrm{R}}\right)}\right),
$$

and experimentally confirmed by Chen et al. ${ }^{11}$ The magnitude of these volume exclusion forces for the polystyrene microspheres and the Au nanoparticles are $35 \mathrm{fN}$ and $9 \mathrm{fN}$, respectively.

The looping of the DNA is driven by thermal fluctuations that provide an energy $k_{\mathrm{B}} T$ to bend and distort the DNA, thereby increasing its entropy while the minimal length scale over which it is possible to form a loop is at least a persistence length $l_{\mathrm{p}}$. These two quantities can be combined to yield a characteristic force scale of only $k_{\mathrm{B}} T / l_{\mathrm{p}} \approx 80 \mathrm{fN}$. Therefore, excluded-volume forces that are smaller than this may be expected to play a concomitantly small role in affecting the kinetics of loop formation. A detailed calculation using the formalism proposed by Blumberg et al. ${ }^{12}$ supports this assertion by suggesting a two-fold decrease in the loop formation rate from the Au nanoparticle to the polystyrene microsphere experiment. This is roughly in line with the observed factor of 1.3. The effect of excluded-volume forces on loop breakdown, however, is expected to be even smaller and would favor faster breakdown in the presence of the bigger microsphere, so cannot account for the observed breakdown data.
Another constraint that is imposed on the DNA in TPM experiments is that the DNA is typically attached to the cover glass and bead through flexible carbon linkers, which act as freely rotating swivel joints. This flexible attachment changes the number of thermal modes available to the DNA when compared with free or infinitely long DNA. ${ }^{12}$ One might argue that the boundary condition at the probe end of the DNA differs between the two experiments, with the nanoparticle allowing the tagged end of the DNA to freely fluctuate while the large microsphere confines the orientation of the DNA to roughly a half plane, similar to the boundary condition at the cover glass. The extra constraint imposed by the large microsphere leads to a decrease in entropy that would tend to stretch the DNA, altering the loop formation and breakdown rates in a similar manner to the excluded volume forces previously discussed. This effect, therefore, also cannot account for the change observed in the loop breakdown rate.

\section{Hydrodynamic Effects}

The effects considered in the last section all fall under the category of potential effects since they each somehow restrict the conformational space of the DNA. In this section, however, we turn our attention to diffusion effects on the looping rate. The proximity of the bead to the cover glass may have an appreciable influence on the loop breakdown kinetics through long-range hydrodynamic interactions as non-slip boundary conditions for fluid flow near a surface increase the hydrodynamic friction. This could provide an explanation for the two-fold increase in the lifetime of the looped state for DNA attached to a polystyrene bead as opposed to a Au nanoparticle. To break a loop the binding energy of the LacI protein to the operator, ${ }^{13} E_{\mathrm{B}} \approx 10^{-19} \mathrm{~J}$, must be overcome by thermal fluctuations. We may assume that dissociation occurs at a rate ${ }^{14}$

$$
k_{\mathrm{R}} \propto \frac{D}{\pi k_{\mathrm{B}} T} e^{E_{\mathrm{B}} / k_{\mathrm{B}} T},
$$

where $D$ is the diffusion constant of the DNA. Since $D \propto$ $1 / \eta$, where $\eta$ is the effective viscosity of the medium, the looping lifetime depends linearly upon the viscosity. As hydrodynamic friction increases in the presence of boundary walls, the thermal fluctuations of the DNA slow down, making dissociation less likely. To quantitatively estimate how hydrodynamic effects might affect the looping lifetime, we can approximate the RMS distance $Z_{\mathrm{RMS}}=\sqrt{\left\langle Z^{2}\right\rangle}$ between the attachment point of the DNA to the bead and the cover glass in the experiment. Segall et al. ${ }^{8}$ provide an approximate formula for $\left\langle Z^{2}\right\rangle$ that includes the excluded volume repulsion 


$$
\left\langle Z^{2}\right\rangle=\left(6-\frac{4}{\sqrt{\pi}} \frac{N_{\mathrm{R}} e^{-N_{\mathrm{R}}^{2}}}{\operatorname{erf}\left(N_{\mathrm{R}}\right)}\right)\left(\frac{l_{\mathrm{c}} l_{\mathrm{p}}}{3}\right) .
$$

From this relation we find $Z_{\mathrm{RMS}}^{\mathrm{PS}} \approx 126 \mathrm{~nm}$ for the polystyrene microsphere and $Z_{\mathrm{RMS}}^{\mathrm{Au}} \approx 110 \mathrm{~nm}$ for the Au nanoparticle when we use $l_{\mathrm{c}}=160 \mathrm{~nm}$ as the length of the DNA in the looped state. Since $R_{\mathrm{B}}^{\mathrm{PS}} \gg Z_{\mathrm{RMS}}^{\mathrm{PS}}$ we may reasonably assume that the microsphere confines the DNA below itself much in the same way as the DNA is confined above the cover glass. An approximate way to calculate the hydrodynamic friction experienced by the DNA is to model this system as that of a spherical object diffusing between two planes, a problem that has been solved exactly in hydrodynamic theory. ${ }^{15,16}$ The situation is somewhat complicated by the fact that the friction coefficient is no longer isotropic, but has different in-plane and axial components, so we will consider each component separately. We should note that similar, but weaker dual-surface hydrodynamic forces will affect the unlooped state. In that instance, the distance between the DNA and the coverslip is increased, and the loop-forming section of the DNA is comparatively far removed from the surfaces. Hence, we will focus our analysis of hydrodynamic effects here on the looped state. To understand how hydrodynamic effects can lead to a two-fold increase in the friction on the DNA, and therefore change the lifetime of the loop accordingly, we compare the case of DNA diffusing or fluctuating near a single surface ${ }^{17,18}$ to that of the same DNA diffusing between two surfaces (see Figure 3). These two cases are representative of the Au nanoparticle and polystyrene microsphere systems, respectively.

We first consider the case of surface tethered DNA attached to a Au nanoparticle. For this simple approximation we will treat the DNA as a sphere of effective radius $a$. The drag coefficient for the lateral motion of a sphere above a plane surface is approximated by Faxen's law ${ }^{17}$

$$
\gamma_{\|}=\frac{\gamma_{0}}{1-\frac{9}{16} x+\frac{1}{8} x^{3}-\frac{45}{256} x^{4}-\frac{1}{16} x^{5}}
$$

and an interpolation formula, ${ }^{18}$ to the same order, for the perpendicular drag coefficient is given by

$$
\gamma_{\perp}=\frac{\gamma_{0}}{1-\frac{9}{8} x+\frac{1}{2} x^{3}-\frac{57}{100} x^{4}-\frac{1}{5} x^{5}} .
$$

In the above, $x=a / b, b$ is the distance between the center of the DNA and the surface, and $\gamma_{0}=6 \pi \eta a$ is the Stokes drag with viscosity $\eta$. Likewise, for the case of surface tethered DNA attached to a large microsphere, we must consider the parallel and perpendicular drag coefficients for the hydro-

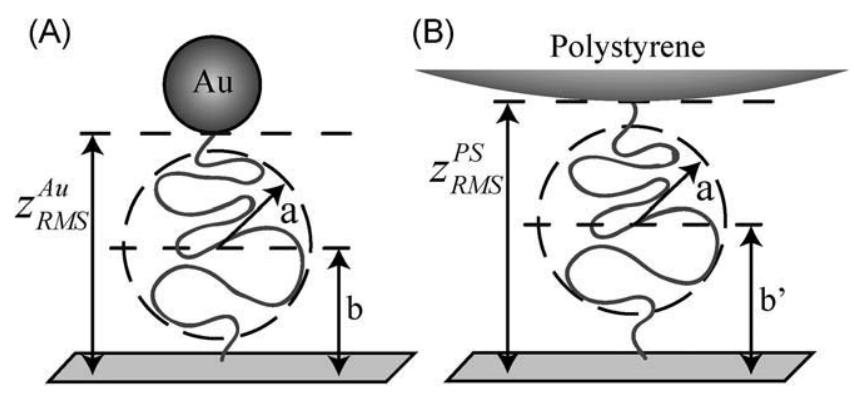

FIGURE 3 Hydrodynamic surface effects on the DNA. A: DNA tethered by a gold nanoparticle is modeled by a sphere of effective radius at a distance $b=Z_{\mathrm{RMS}}^{\mathrm{Au}} / 2$ from a planar surface. B: DNA tethered to a large polystyrene microsphere is modeled by DNA of the same effective radius $a$, at a distance $b^{\prime}=Z_{\mathrm{RMS}}^{\mathrm{PS}} / 2$ between two parallel planes.

dynamic motion of a sphere between two parallel surfaces, $\gamma_{\|,}^{\prime} \gamma_{\perp}{ }_{\perp}$. We do not have a simple interpolative formula for these coefficients, nonetheless, the values can be extracted from the numerical results reported by Ganatos et al. ${ }^{15,16}$

We next assume that the center of the DNA lies midway between the bead and the coverslip and we take this distance to be $b=Z_{\mathrm{RMS}}^{\mathrm{Au}} / 2$ and $b^{\prime}=Z_{\mathrm{RMS}}^{\mathrm{PS}} / 2$ for the Au nanoparticles and the polystyrene microspheres respectively. If we assume that the effective radius of the DNA does not change, it follows that an effective radius for the DNA of $a=0.57 b$ ' for in-plane motion and $a=0.73 b$ ' for axial motion would lead to a friction coefficient of the DNA that is twice as large for the polystyrene microsphere experiment as for the Au nanoparticle experiment.

These values, which are similar in size, give reasonable estimates of 36-46 $\mathrm{nm}$ for the effective hydrodynamic radius of the DNA segment responsible for loop breakdown. While this simple model does not prove that hydrodynamic effects on the diffusive behavior of the DNA give rise to the observed difference in looped lifetimes, the previous analysis does show that such effects are sufficiently large. Moreover, such hydrodynamic effects should have little effect on the protein due to its small size, which supports the notion that fluctuations in the DNA, as opposed to the protein, are the primary cause for the breakdown of protein-mediated DNA loops.

\section{CONCLUSIONS}

We have shown that the attached microsphere in TPM experiments affects measured loop formation and breakdown rates in the lactose repressor system only within a factor of two. As this system is exceptionally sensitive to mechanical forces and constraints, we can confidently state that measured kinetic parameters like binding and dissociation con- 
stants or motor protein velocities can be accurately measured quantitatively by TPM within those limits. We further attribute bead effects primarily to hydrodynamic interactions between the bead, cover slip, and the biomolecule under study.

\section{MATERIALS AND METHODS}

For the TPM experiments, we synthesized a 790 bp end-labeled dsDNA fragment containing two LacI-binding operator sites $320 \mathrm{bp}$ apart by polymerase chain reaction (PCR). The DNA is tagged with either $\mathrm{Au}$ nanoparticles or polystyrene microspheres and is then tethered to a microscope cover glass within a sample chamber containing a $200 \mathrm{pM}$ solution of LacI protein.

\section{Tethering Protocol}

The PCR primers are modified with biotin and digoxigenin. The end-labeled DNA is incubated with either streptavidin coated $\mathrm{Au}$ nanoparticles (Nanocs Inc.) radius $R_{\mathrm{B}}=25 \mathrm{~nm}$, or streptavidin coated polystyrene microspheres (Spherotech) $R_{\mathrm{B}}=400 \mathrm{~nm}$, with relative concentrations of approximately $1: 10$, in a $50 \mu \mathrm{l}$ volume of PTC1 buffer $(20 \mathrm{mM}$ Tris-Acetate, $\mathrm{pH} 8.0,130 \mathrm{mM} \mathrm{KCl}, 4 \mathrm{mM}$ $\mathrm{MgCl}_{2}, 0.1 \mathrm{mM}$ EDTA, $0.1 \mathrm{mM}$ DTT, $20 \mu \mathrm{g} / \mathrm{ml} \mathrm{BSA}, 80 \mu \mathrm{g} / \mathrm{ml}$ Heparin, $1 \mathrm{mg} / \mathrm{ml} \alpha$-casein). The excess of beads prevents the formation of multiple DNA tethers. The DNA-bead mixture is incubated for $30 \mathrm{~min}$ at $5^{\circ} \mathrm{C}$ on a rotating rack. Sample chambers consisting of a microscope slide, a cover glass, and a parafilm spacer are washed with $200 \mu$ l phosphate buffered saline (PBS) buffer $(137 \mathrm{mM} \mathrm{NaCl}$, $10 \mathrm{mM}$ Phosphate, $2.7 \mathrm{mM} \mathrm{KCl}$, pH 7.4) and then filled with $20 \mu \mathrm{g} /$ $\mathrm{ml}$ anti-digoxigenin (polyclonal from sheep, Roche Scientific) to coat the slide with antibodies. Excess antibodies in the solution are washed away with $200 \mu$ PTC1. Continued incubation with PTC1 for half an hour blocks the surface against nonspecific binding. The DNA-bead mixture is then pipetted into the sample chamber and incubated for $5 \mathrm{~min}$. The excess beads are washed away with $400 \mu \mathrm{l}$ PTC1. The buffer is replaced with LBB buffer ( $10 \mathrm{mM}$ Tris- $\mathrm{HCl}, \mathrm{pH}$ 7.4, $200 \mathrm{mM} \mathrm{KCl}, 0.1 \mathrm{mM}$ EDTA, $1 \mathrm{mg} / \mathrm{ml} \alpha$-casein, $0.05 \mathrm{v} / \mathrm{v}$ DMSO, $0.2 \mathrm{mM}$ DTT) by pipetting $200 \mu \mathrm{l}$ into the chamber. Finally, the buffer is entirely replaced by introducing $100 \mu \mathrm{l}$ of a $200 \mathrm{pM}$ solution of LacI protein in LBB into the chamber.

\section{Imaging and Analysis}

The tethered nanoparticles are imaged by darkfield microscopy on a Zeiss Axiovert S100 inverted microscope that is custom fitted with an Olympus U-DCW darkfield condenser (oil, N.A. 1.2-1.4) and a $100 \times$ Olympus UPlan-FLN (oil, N.A. 0.6-1.3) darkfield objective. The polystyrene microspheres are imaged by brightfield microscopy using the same objective. All images are acquired at $30 \mathrm{~ms}$ intervals on a Cascade 650 cooled CCD camera (Photometrics). At this frame rate the influence of time averaging by the detector is negligible. ${ }^{19}$ The particles are tracked with custom MATLAB software that implements a Gaussian mesh algorithm to locate the center of each nanoparticle. ${ }^{20}$ With this algorithm we are able to determine the beads center with an error of less than $5 \mathrm{~nm} .{ }^{12}$ The resulting radial coordinates are then filtered with a $0.05 \mathrm{~Hz}$ Butterworth filter to remove slow drift.
Figure 2 provides an example of the data obtained from measurements with $\mathrm{Au}$ nanoparticles and polystyrene microspheres. Loop formation and breakdown events are readily visible in the time traces. The looped and unlooped states are also quite evident in the double-peaked histograms of the motion of the markers. The lifetimes of the looped and unlooped states can be extracted from these traces by performing a running average over the xy motion of the bead and then using a simple thresholding algorithm to detect looping and unlooping of the DNA. By fitting Gaussians to the looped and unlooped distributions displayed in the histogram, the threshold was chosen at the minimum between the peaks of the two distributions. The resulting rates were fairly insensitive to the exact location of the threshold; a $10 \%$ shift in the threshold from the fitted location resulted in only a $<5 \%$ change in the kinetic rates. We also note that the quality of the traces is slightly better for the $\mathrm{Au}$ nanoparticle data; the extracted kinetic rate constants are, however, insensitive to differences in noise at that level. Data was acquired for $20 \mathrm{~min}$ for each of 7 tethered Au nanoparticles and 7 tethered polystyrene microspheres. Slight quantitative differences in the looping and unlooping rates, of roughly $10-15 \%$, where found when we varied the window size from 1 to $3 \mathrm{~s}$.

The authors would like to thank Brian Lewis and Justin Blaty for helping with the TPM experiments and Jason Kahn, from the University of Maryland, for providing the LacI protein.

\section{REFERENCES}

1. Nelson, P. C. Curr Opin Colloid Interface Sci 2007, 12, 307-313.

2. Olympus Microscopy Resource Center: Introduction to Microscopy. Available at: http://www.olympusmicro.com/primer/index. html (accessed May 17, 2010).

3. Kato, H.; Nishizaka, T.; Iga, T.; Kinosita, K Jr.; Ishiwata, S. Proc Nat Acad Sci 1998, 96, 9602-9606.

4. Finzi, L.; Gelles, J. Science 1995, 267, 378-380.

5. Vanzi, F.; Broggio, C.; Sacconi, L.; Pavone, F. S. Nucleic Acids Res 2006, 34, 3409-3420.

6. Sperling, R. A.; Gil, P. R.; Zhang, F.; Zanella, M.; Parak, W. J. Chem Soc Rev, 2008, 37, 1896-1908.

7. Chen Y. F.; Milstein, J. N.; Meiners, J.-C. Phys Rev Lett 2010, $104,048301$.

8. Segall, D. E.; Nelson, P. C.; Phillips, R. Phys Rev Lett 2006, 96, 088306.

9. Wong, O. K.; Guthold, M.; Erie, D. A.; Gelles, J. PLoS Biol 2008, 6, e232.

10. Han, L.; Garcia, H. G.; Blumberg, S.; Towles, K. B.; Beausang, J. F.; Nelson, P. C. PLoS ONE 2009, 4, e5621.

11. Chen, Y.-F; Wilson, D. P.; Raghunathan, K.; Meiners, J.-C. Phys Rev E 2009, 80, 020903(R).

12. Blumberg, S.; Gajraj, A.; Pennington, M. W.; Meiners, J.-C. Biophys J 2005, 89, 1272-1281.

13. Hsieh, W. T.; Whitson, P. A.; Matthews, K. S.; Wells, R. D. J Biol Chem 1987, 262, 14583-14591.

14. Gardiner, C. Handbook of Stochastic Methods: For Physics, Chemistry and the Natural Sciences; Springer-Verlag: Berlin, 2004. 
15. Ganatos, P.; Weinbaum, S.; Pfeffer, R. J Fluid Mech 1980, 99, 739-753.

16. Ganatos, P.; Pfeffer, R.; Weinbaum, S. J Fluid Mech 1980, 99, 755-783.

17. Happel, J.; Brenner, H. Low Reynolds Number Hydrodynamics. With Special Applications to Particulate Media; Noordhoff International Publishing: Leyden, 1983.

18. Schaffer, E.; Norrelykke, S. F.; Howard, J. Langmuir 2007, 23, 3654-3665.
19. Han, L.; Lui, B.; Blumberg, S.; Beausang, J. F.; Nelson, P. C. In Mathematics of DNA Structure, Function, and Interactions; Benham, C. J.; Harvey, S.; Olson, W. K.; Sumners, D. W.; Swigon, D., Eds.; IMA Volumes in Mathematics and its Applications; Springer: New York, 2009, 150, pp 123-138.

20. Thompson, R.; Larson, D.; Webb, W. Biophys J 2002, 82, 2775 2783.

Reviewing Editor: Sarah A. Woodson 